\title{
Omnidirectional bearing-only see-and-avoid for small aerial robots
}

\author{
Luis Mejias* \\ luis.mejias@qut.edu.au
}

\begin{abstract}
Motivated by the growing interest in unmanned aerial system's applications in indoor and outdoor settings and the standardisation of visual sensors as vehicle payload. This work presents a collision avoidance approach based on omnidirectional cameras that does not require the estimation of range between two platforms to resolve a collision encounter. It will achieve a minimum separation between the two vehicles involved by maximising the view-angle given by the omnidirectional sensor. Only visual information is used to achieve avoidance under a bearing-only visual servoing approach. We provide theoretical problem formulation, as well as results from real flight using small quadrotors.
\end{abstract}

\section{INTRODUCTION}

The growing interest in Unmanned Aerial System (UAS) applications in indoor and outdoor setting is motivating a great interest in industry and research community [1],[2],[3]. With many groups working already with small UAS and adopting these platforms as preferable testbed, is it expected that a new set of regulations will appear aiming to define minimum capabilities and compliance terms for small aerial robotic systems. In fact, in 2004 a set of standards for see and avoid was released [4], serving as guidelines for developers and researchers working on UASs. In 2005, United States Department of Defence (DoD) adopted these performance standards for its UAS program. With these regulation already in place and the acknowledgement that UAS collision avoidance "see-and-avoid" is one of the most significant challenges facing the integration of UASs into the national airspace [5], this paper present a contribution to the field of see-and-avoid based on omnidirectional visual sensors.

Small UASs have inherent limitations in payload and available onboard power making vision and other lightweight sensors a very attractive alternative. Vision as a sensor not only offers a rich source of information for navigational purposes, but it can also offer the best chances for regulator approval [6]. The conventional means of resolving a collision encounter between two of more aerial vehicles is through the use of cooperative approaches that in most cases involve range estimation. Shakernia et al. developed a technique that estimates range by manoeuvring the owncraft finding that range converge in 7-15 secs [7](passive ranging).

Driessen proposed an approach using monocular cameras that uses bearing-rate combined with target growth rate

*The author is with the Australian Research Centre for Aerospace Automation (ARCAA). Queensland University of Technology, QLD 4001, Australia

**The authors are with Computer Vision Group, Centro de Automática y Robótica CAR, Universidad Politécnica de Madrid, C. José Gutiérrez Abascal 2, 28006 Madrid, Spain

\author{
Iván F. Mondragón Pascual Campoy** \\ if.mondragon@upm.es, \\ pascual.campoy@upm.es
}

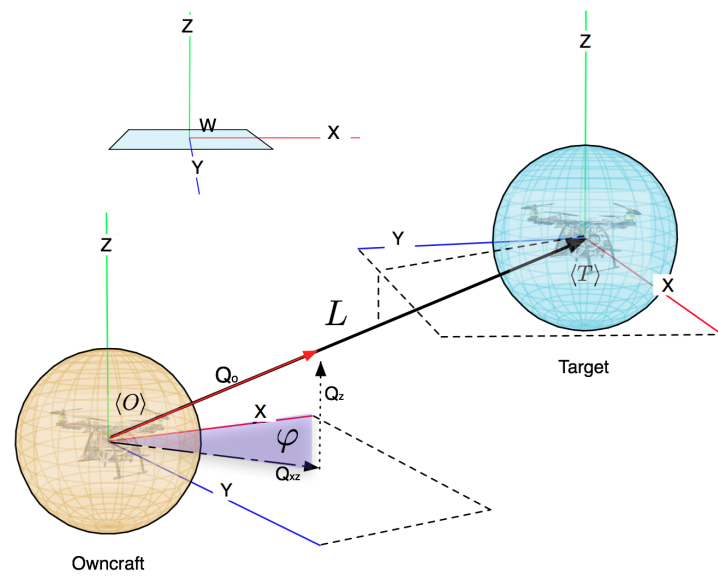

Fig. 1. Owncraft-Target setup in 3D. Each vehicle is enclosed inside a sphere of radius $\mathrm{R}$ that correspond to the minimum volume occupied by the vehicle. By maximising $\varphi$, we can guarantee that $\mathrm{L}$ will be higher than twice the radius of the sphere that contains each vehicle

in the image to estimate distance [8]. Fasano combined cameras and pulsed radar (to obtain range) to propose an approach for non-cooperative scenarios [9]. Saunders and Beard [10] developed an approach for fixed-wing vehicles to avoid obstacles using image measurements similar to the approach presented here but their approach uses range to objects. Once more, Watanabe et al. [11] proposed a PNbased guidance law defining a collision cone using vision sensors, but their assumptions about knowledge of obstacle location may be difficult to validate in real flights, in the context of monocular vision. Similarly, Angelov et al. [12] have proposed a passive approach to collision avoidance assuming EO sensors. However, their bearing-rate approach presents many ambiguities when more than two scenarios are considered. Mejias et al. [13] and Lai et al. [14] have done some pioneering work in the detection and conflict resolution using real flight test with manned and unmanned platforms.

Predominately so far, the trend has been to use range or minimum separation as main or complementary information source to resolve collision conflicts. This paper present a important contribution based on omnidirectional sensors that do not require explicit knowledge of obstacle range to avoid them. Also, we contribute with a visual servoing approach using omnidirectional sensors for aerial robots in real flights. We propose that the minimum separation between a vehicle 
and a target is achieved by maximising the view-angle $(\varphi)$ given by the omnidirectional sensor (Figure 1).

This paper is structured as follows. Section II presents the problem statement. Section III introduces the camera projection model and tracking approach. Section IV presents the experimental setup and algorithm evaluation in real flight tests on a small quadcopter. Finally, conclusions and future work are presented.

\section{PRoblem DESCRIPTION}

This section presents the vehicle kinematic model and the formulation of our avoidance problem. This paper considers a vehicle moving with constant altitude, therefore the kinematic of each vehicle is abstracted as an unicycle model. Next, we describe the details of these two approaches.

\section{A. Vehicle model}

Let a Cartesian navigation frame $\left(x_{n}, y_{n}, z_{n}\right)$ have origin at the location of a vehicle position when a target is detected at $t=0$ (Figure 2). Assume the vehicle has constant ground speed $v$ then its translational motion in the navigation frame can be described by

$$
\dot{x}_{n}=v \cos \psi, \quad \dot{y}_{n}=v \sin \psi, \quad \dot{\psi}=\omega
$$

where $\left(x_{n}, y_{n}\right) \in \Re^{2}$ is the vehicle location and $\psi \in(-\pi, \pi)$ its heading. This model is constrained by the vehicle's maximum turning rate and linear velocity, i.e, $v_{\min }<$ $v<v_{\max }$ and $|\omega|<\omega_{\max }$, respectively. The two vehicles involved in the scenario are owncraft (O) (vehicle with camera) and target ( $\mathrm{T}$ ) (vehicle to avoid or static target). The control inputs of (O) and (T) are linear and angular velocities $\left[\begin{array}{ll}v_{O} & \omega_{O}\end{array}\right]^{T}$ and $\left[\begin{array}{ll}v_{T} & \omega_{T}\end{array}\right]^{T}$, respectively. Where $v=\left[\begin{array}{lll}v_{x} & v_{y} & v_{z}\end{array}\right]^{T}$ and $\omega=\left[\begin{array}{lll}\omega_{x} & \omega_{y} & \omega_{z}\end{array}\right]^{T}$.

The view angle (or relative bearing) is denoted $\varphi$. This angle is given by the onboard omnidirectional sensor (described in the next section).

\section{B. Problem formulation}

With regards to Figure 2, the whole system kinematics can be written as follows:

$$
\dot{s}=J(s, L) u
$$

where $s=\left[\begin{array}{ll}\phi & \varphi\end{array}\right]^{T}$ is the feature vector with $\phi$ and $\varphi$ being the elevation and bearing of the target wrt owncraft ${ }^{1}$, $u=\left[v_{O} \omega_{O}\right]$ as defined above and $J(s, L)=J(s, L)_{\text {trans }}+$ $J(s)_{\text {rot }}$ the jacobian which can be decomposed in a translational and rotational part (see [15], [16] for more details). Therefore, (2) can be expressed in a translational and rotational component. Let us define the control task such as

$$
u=J(s, L)^{+} \dot{s}
$$

where $\dot{s}=-\lambda\left(s-s^{*}\right)$ is the error signal, with $\lambda>0, s^{*}$ the desired feature value and $J(s, L)^{+}$the pseudo-inverse [17],

\footnotetext{
${ }^{1}$ note: we assume that camera and vehicle $\mathrm{cg}$ are aligned, approximately in the same place. Also, the sensor provides $\phi$ and $\varphi$, but only the use of $\varphi$ is explored in this paper
}

[18]. The aim is to minimise the error signal by maximising the desired value of our features $\left(\varphi \rightarrow \pm \frac{\pi}{2}\right)$. This is achieved using visual servoing approach [17]. The details are presented in section IV.

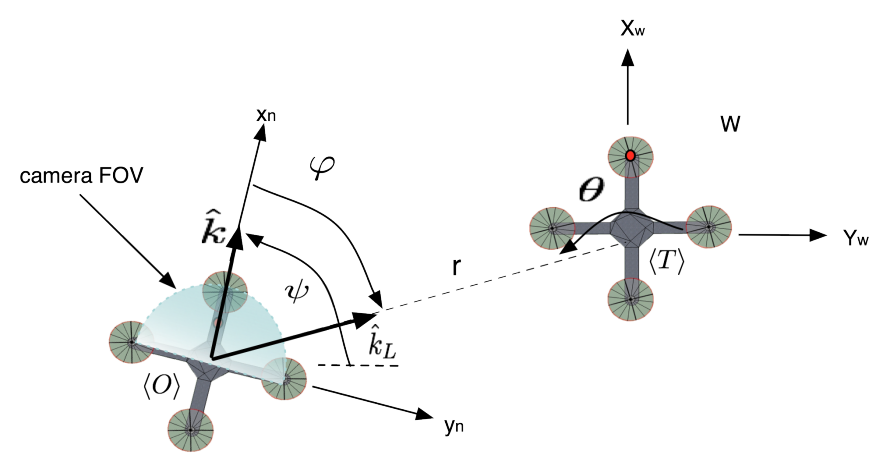

Fig. 2. Owncraft-Target setup in 2D.

Our hypothesis is: 1) that collision avoidance is achieved when L (see Figure 1) is higher than twice the radius of the sphere that contains each vehicle $(L>2 R)$. Furthermore, $\mathrm{L}$ is maximum when $|\varphi|$ is maximum. We achieve minimum separation between a vehicle and a target by maximising the view angle given by the omnidirectional sensor. 2) that collision avoidance is achieved by following a spiral that results from maintaining a fixed-relative bearing to the target until a stopping condition is reached.

Using a similar development as presented in [19] and using the vehicle kinematic described by (1) expressed in polar coordinates

$$
\begin{aligned}
& x_{w}(t)=r(t) \cos (\theta(t)) \\
& y_{w}(t)=r(t) \sin (\theta(t))
\end{aligned}
$$

with time $t$ and polar coordinates $r, \theta$.

We can demonstrate that the trajectory followed by our vehicle will be described by the following equation

$$
r(u)=a e^{b u}
$$

with $a=r_{0}, b=\cot (\varphi)$ and $u=\theta_{0}-\theta$ which is the equation for an equiangular spiral in polar coordinates [19].

In the case that a stopping condition in the vehicle heading is defined (such as $f\left(\psi^{*}\right) \in[-\pi, \pi]$ ), the vehicle will avoid the collision keeping a minimum distance $r^{*}$. A stopping condition can be define as $\theta^{*}=f\left(\psi^{*}\right) \in[-\pi, \pi]$ and $r^{*}$ is the closest distance to target at $\psi^{*}$. Because $r$ is always decreasing as $t \rightarrow \infty, r^{*}$ is the closest range of approach to the object for stopping condition $\theta^{*}=f\left(\psi^{*}\right)$ and with $b \neq 0$ proves that as long as the detection occurs at a range $L>2 R$, it will avoid the target with $r^{*}>2 R$.

This theorem can be extended for moving targets. In this case it will result in a large set of spirals for the general case of which (5) is a special case. 


\section{Detection ApProACh}

This paper considers an image pre-processing approach that transforms the input image into a RGB normalised colour space [20] followed by a Continuously Adaptive Mean Shift (Camshift) [21] approach to detect and track persistent features (targets).

\section{A. Camera model}

Omnidirectional sensing can be achieved with catadioptric or dioptric systems. Here, we have implemented our approach using a dioptric system (fisheye camera). For more details on catadioptric or dioptric systems see [22], [23]. Ying and $\mathrm{Hu}$ have demonstrated previously that it is reasonable to assume that the small projection locus can be approximated by a single viewpoint [24], allowing to extend the unify model presented below, to fisheye cameras.

We based our model in the developments made by Geyer and Daniilidis [23] and Barreto and Araujo [25]. Figure 3 shows the general unit sphere projection for modelling catadioptric systems.

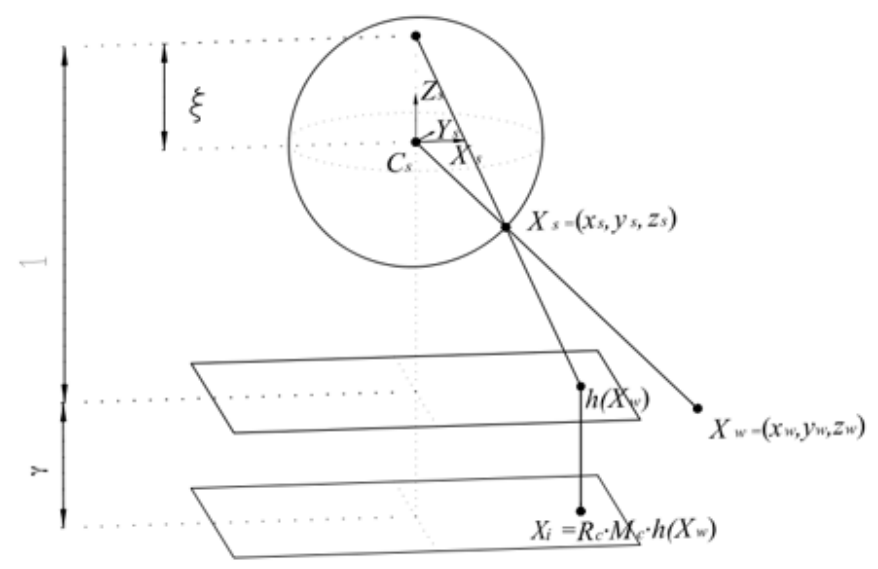

Fig. 3. Catadioptric projection modelled by the unit sphere.

Let us consider a point in space (visible to the catadioptric system), with Cartesian coordinates $X_{w}=\left(x_{w}, y_{w}, z_{w}\right)^{T}$ in the catadioptric reference (focus). This point is mapped into point $X_{s}=\left(x_{s}, y_{s}, z_{s}\right)^{T}$ on the unitary sphere centred on the effective view point by $X_{s}=X_{w} / \sqrt{x_{w}^{2}+y_{w}^{2}+z_{w}^{2}}=$ $X_{w} /\left\|X_{w}\right\|$. To each point $X_{s}$ in the unit sphere corresponds a projective point $X_{c}=\left(x_{c}, y_{c}, z_{c}\right)^{T}$ in a coordinate system with origin at the camera projection centre. This projection is a non-linear mapping between two projective planes and is defined by (6).

$$
X_{c}=\left(x_{c}, y_{c}, z_{c}\right)^{T}=M_{c} \cdot \hbar\left(X_{w}\right)
$$

with

$$
\begin{aligned}
& M_{c}=\left[\begin{array}{ccc}
\gamma & \gamma \alpha & u_{0} \\
0 & r \gamma & v_{0} \\
0 & 0 & 1
\end{array}\right] \\
& \hbar\left(X_{w}\right)=\left(x_{w}, y_{w}, z_{w}+\xi \sqrt{x_{w}^{2}+y_{w}^{2}+z_{w}^{2}}\right)^{T}
\end{aligned}
$$

where $\xi$ is the parameter that define the projection of a point on the unitary sphere into the normalised plane $Z=$ $1-\xi$ (depends of the type of mirror of lens used), and the matrix $M_{c}$ is the camera projection matrix (where $\gamma$ is the generalised focal length, $\left(u_{0}, v_{0}\right)$ is the principal point, $\alpha$ the skew and $r$ is the aspect ratio).

Finally, the image in the catadioptric plane is obtained after a collineation between the image and the projective plane $\left(H_{c}=R_{c} \cdot M_{c}\right)$, depending on the rotation of the camera $R_{c}$. The projection of a world point on the catadioptric image is defined by $X_{i}=H_{c} \cdot \hbar\left(X_{w}\right)$.

The function $\hbar\left(X_{w}\right)$ is homogenous positive injective with inverse $\hbar^{-1}\left(X_{w}\right)$. This function map points in the projective plane onto the unitary sphere. The non-linear inverse function is defined by (7).

$$
\begin{aligned}
\left(x_{s}, y_{s}, z_{s}\right)^{t} & =\hbar^{-1}\left[H_{c}^{-1} X_{i}\right] \\
& =\left(\lambda_{c} x_{c}, \lambda_{c} y_{c}, \lambda_{c} z_{c}-\xi\right)^{T}
\end{aligned}
$$

where $\lambda_{c}=\frac{\left(z_{c} \xi+\sqrt{z_{c}^{2}+\left(1-\xi^{2}\right)\left(x_{c}^{2}+y_{c}^{2}\right)}\right)}{\left(x_{c}^{2}+y_{c}^{2}+z_{c}^{2}\right)}$

In our implementation, we used a dioptric system (fisheye camera). The calibration of the camera was performed using the method described in [26], obtaining the parameter $\xi$ and the matrix $M_{c}$. Figure 4 shows the dioptric system employed and the projection of a captured image on the unitary sphere using the calibration data.

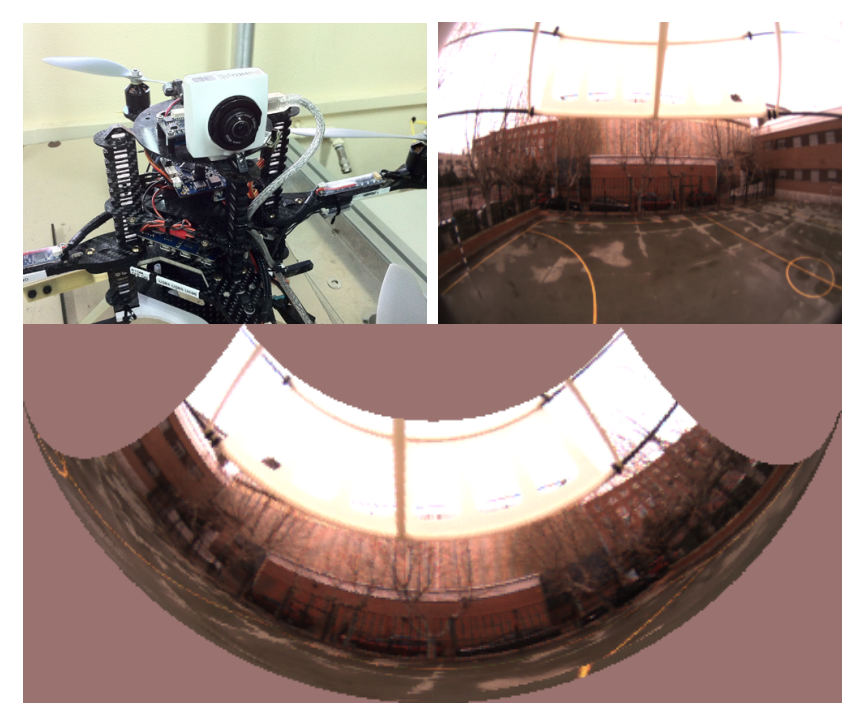

Fig. 4. Omnidirectional system used. Top Left: The dioptric system employed. Top Right: Omnidirectional image taken with this system. Bottom: Projection on the unitary sphere of the omnidirectional image.

\section{B. Colour space and target tracking}

Our focus is primarily to implement the colour space that best maintain object's apparent colour in outdoor settings as seen by a machine vision camera. We tested several colour 
[20], finding that normalised RGB preserves better object's colour in outdoor setting.

We approach the problem of tracking by exploiting the colour characteristic of the target. We define a basic colour (blue) to the target by attaching a simple coloured mark to it and tracking this mark. We then use an algorithm that has proven to deal with outdoor lighting changes by dynamically adapting to changes in probability distributions, the Continuously Adaptive Mean Shift [21] (CamShift). This algorithm is based in the mean shift originally introduced by Fukunaga and Hostetler [28] (see Figure 5).

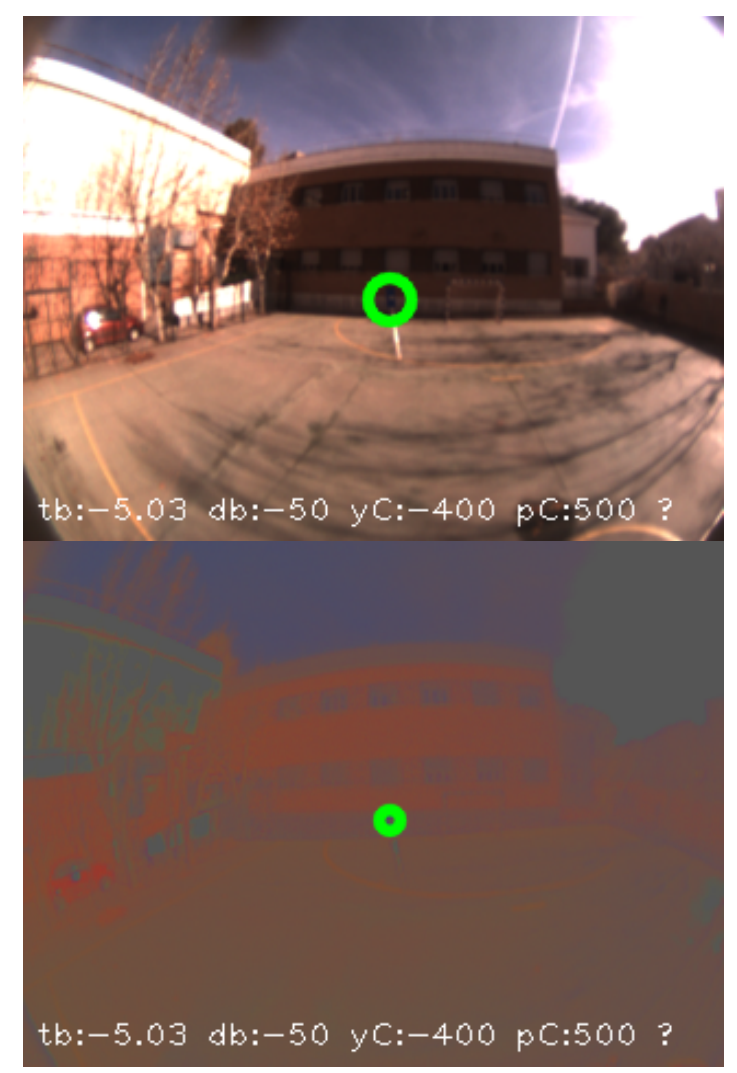

Fig. 5. Top: Angle estimation on the owncraft. Angle $\varphi$ (tb in the image) is the relative bearing to the target. Bottom: Result of tracking in corresponding normalised RGB image using camshift algorithm. In this Figure, tb is target bearing, $\mathrm{db}$ is desired target bearing, $\mathrm{yC}$ is yaw command and $\mathrm{pC}$ is pitch command

\section{Target attitude estimation}

The CamShift algorithm is used to track a target in the original images, obtaining at each iteration the image coordinates of the target point $X_{T_{c}}=\left(x_{t_{c}}, y_{t_{c}}, 1\right)^{T}$. The coordinates of the target point in the image plane are back projected on the unitary sphere model using (7), obtaining the target projection point in the unitary sphere $X_{t_{s}}=$ $\left(x_{t_{s}}, y_{t_{s}}, z_{t_{s}}\right)^{T}$. Finally, (8) is used to obtain the target attitude wrt vehicle. This equation already includes the rotation between the camera unitary sphere and the aircraft coordinate systems.

$$
\phi_{t}=\arctan \left(\frac{y_{t_{s}}}{z_{t_{s}}}\right) ; \varphi_{t}=\arctan \left(\frac{x_{t_{s}}}{z_{t_{s}}}\right)
$$

where $\phi_{t}$ and $\varphi_{t}$ represent the target elevation and bearing as seen by our omnidirectional camera. $\varphi_{t}$ is measured from the camera optical axis which coincides with vehicle longitudinal $x$ axis. Clockwise $\varphi_{t}>0, \varphi_{t} \in\left[0, \frac{\pi}{2}\right)$ and counterclockwise $\varphi_{t}<0, \varphi_{t} \in\left[0,-\frac{\pi}{2}\right)$.

\section{Control Approach}

In this paper, control is achieved using a two stage control architecture. A low level controller is responsible for attitude stabilization, whilst a high-level vision-based controller sends desired attitude values based on image features, therefore a visual servoing approach is used and defined as follows.

Let's assume the two angles in (8) are obtained from the detection approach. We can then follow a similar approach to that used in [16][17][18][29] to construct the image jacobian (previously introduced in section II-B) using our point feature vector $s=s(\phi, \varphi)$ and the desired $s^{*}=s^{*}\left(\phi^{*}, \varphi^{*}\right)$. However, we anticipate that our image Jacobian will have a similar form to those presented in [16][30]. Recalling (2) and re-writing in a two component form

$$
\dot{s}=J(s, L) u=\left\{J(s, L)_{\text {trans }}+J(s)_{\text {rot }}\right\} u
$$

where each component of $J(s, L)$ is a $2 \times 3$ matrix. The translational part is a function of depth and location of the image features, however the rotational only depends on location. Thus, (9) can be re-written as

$$
\left[\begin{array}{c}
\dot{\phi} \\
\dot{\varphi}
\end{array}\right]=\frac{1}{L} J(s)_{\text {trans }}\left[v_{O}\right]^{T}+J(s)_{\text {rot }}\left[\omega_{O}\right]^{T}
$$

This equation fully relates velocity of features in the image plane with camera (vehicle) linear and angular velocities. The dependency in $\mathrm{L}$ and invertibility of $J$ imposes some constrains in the minimum number of features, type of servoing [31] and knowledge of feature depth. To deal with these constrains the following assumptions are made:

1) Vehicle altitude is kept constant during the experiments. This lead us to approximate $v_{z_{O}}=0$

2) Velocity in $x$ is constant $\left(v_{x}=0.5, \forall t \neq 0\right)$

3) Velocity in $y$ is set to zero initially, i.e, $v_{y}=0, t=0$ however $v_{y} \neq 0, \forall t>0$

4) Vehicle roll and pitch are approximately constant, therefore angular velocities in $x$ and $y$ are negligible. $\omega_{x_{O}} \approx \omega_{y_{O}} \approx 0$

5) Target distance is large enough to consider features at infinity. This lead us to a direct relationship between optical flow and angular velocity. This is possible because of the constant elements 0 and 1 in the jacobian, indicating that elevation is invariant to rotation about the optical axis and relative bearing angle is invariant to optical axis translation.

Taking the vector containing $\dot{\varphi}$ in (10), and rewriting 


$$
\dot{\varphi}=\frac{1}{L}\left[J(s)^{21} J(s)^{22} 0\right]\left[\begin{array}{l}
v_{x_{O}} \\
v_{y_{O}} \\
v_{z_{O}}
\end{array}\right]+\left[J(s)^{21} J(s)^{22} 1\right]\left[\begin{array}{c}
\omega_{x_{O}} \\
\omega_{y_{O}} \\
\omega_{z_{O}}
\end{array}\right]
$$

finally, using our assumptions we can express (11) as

$$
\omega_{z_{O}}=-k_{z}\left(\varphi_{t}-\varphi_{t}^{*}\right)
$$

This control law is used to keep the target at a given relative bearing angle $\varphi_{t}>0$ in the image plane while the vehicle moves with constant velocity.

\section{ApProach Implementation \& Results}

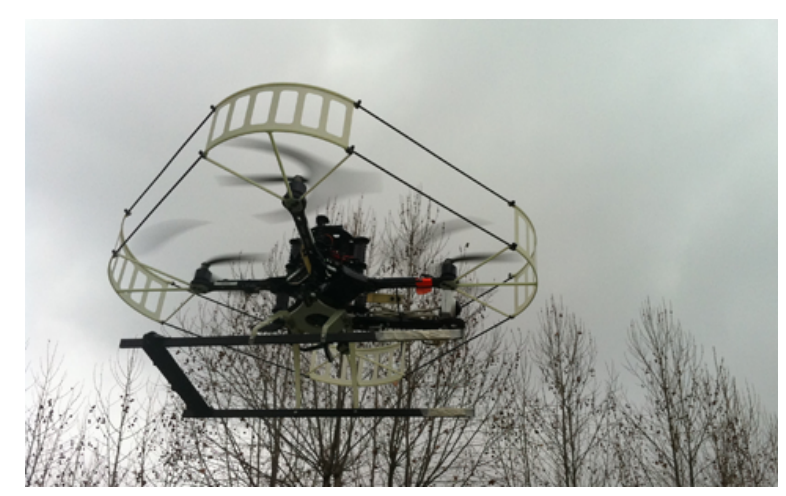

Fig. 6. CVG-UPM [32] Pelican QuadCopter testbed used for sense and avoid experiments

We performed experimental tests using a Pelican quadrotor [33] and a static target (flight trial complexity is greatly reduce if a static target is used initially). The testbed is shown in Figure 6. Onboard vision processing runs on a dual core Atom $1.6 \mathrm{GHz}$ processor with $1 \mathrm{~GB}$ RAM, wireless interface and USB camera. Our vision-based controller communicates via serial interface with the low-level autopilot. This controller sends velocity references to the autopilot creating a dynamic look-and-move servoing architecture at $20 \mathrm{~Hz}$.

Figures 7 to 10 present four of the most representative experiments conducted over several non-consecutive days. All plots show the vehicle trajectory, target location and distance $\mathrm{L}$, where $(\mathrm{O})$ is the starting vehicle position and (X) the target position. Vehicle evasive trajectory depends on initial target detection in the image. It will move left around the target if $\varphi_{0}>0$ setting $\varphi_{t}^{*}=50^{\circ}$, and right around the target if $\varphi_{0}<0$ setting $\varphi_{t}^{*}=-50^{\circ}$.

During these experiments, the vehicle was set to keep a constant longitudinal velocity of $0.5 \mathrm{~m} / \mathrm{s}$ at fixed altitude. In all flights the vehicle starts heading toward the target with constant velocity, when the target is detected and consistently tracked, servoing commands are sent to the autopilot until the experiment stops.

In all cases, we can observe the evolution of the owncrafttarget distance (L). We achieved a minimum separation of $0.8 m t s$ in the test-case depicted in Figure 9. Although not ideal $^{2}$, is expected and enough to achieve successful

\footnotetext{
${ }^{2}$ ideally we would like $\mathrm{L}$ to be as maximum as possible
}

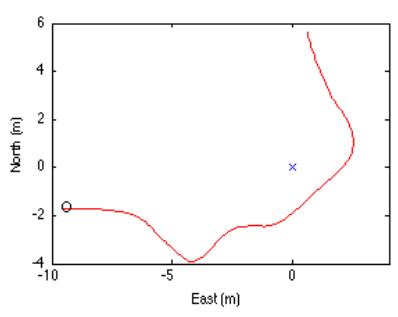

a)

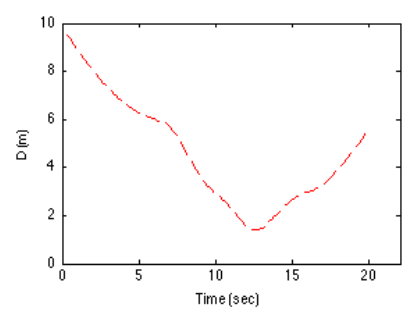

b)
Fig. 7. Experiment 1, run 1. a) shows the vehicle trajectory and location of the target (local tangent plane) where $\mathrm{O}=$ starting location and $\mathrm{X}=$ target location. b) shows the evolution of $\mathrm{L}$ with a minimum value of $1.3 \mathrm{mts}$

avoidance in this case given that our target had a radius of $0.2 m t s$ and our vehicle radius is $0.2 m t s$ approx (therefore, $L>0.4 m t s$ ). We can attribute this not ideal behavior to environmental factors such as wind, which was not considered in simulation stages.

In all other three cases, the minimum separation achieved was $1.3 \mathrm{mts}, 2.34 \mathrm{mts}$ and $2.35 \mathrm{mts}$, respectively. These magnitudes are well above $0.4 \mathrm{mts}$ which corroborates our hypothesis of $L>2 R$.

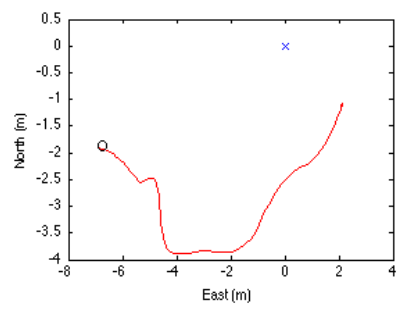

a)

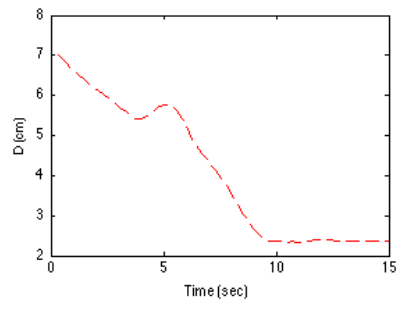

b)
Fig. 8. Experiment 2, run 3.a) shows the evasive trajectory of the vehicle and the location of the target (local tangent plane). b) shows the evolution of $L$ with a minimum value of $2.34 \mathrm{mts}$

Additional experiments can be found in the project web page [32].

\section{CONCLUSION}

This paper presented a robust real time method for UAS see and avoid based only in the visual information provided by an omnidirectional camera sensor. The system was tested on real UAS flights in collision scenarios, showing that the proposed method is an efficient technique for real time evasion. In addition, the proposed hypothesis that maximizing avoidance angle will maximise minimum avoidance distance has been demonstrated in real flights.

Experimental results have validated the proposed approach, showing a good response even if the evasion maneuver starts with a small range to the colliding target. Future work includes testing the approach in a dynamic scenario with two vehicles engaged in collision scenario.

\section{ACKNOWLEDGMENTS}

The work presented in this paper is the result of an ongoing collaboration between the Computer Vision Group - Universidad Politécnica de Madrid and the Australian Research Centre for Aerospace Automation (ARCAA). This 


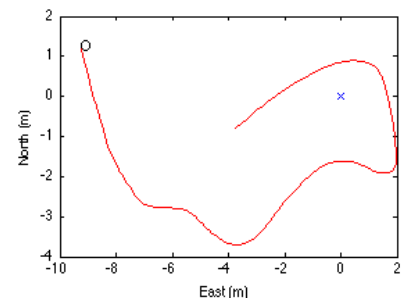

a)

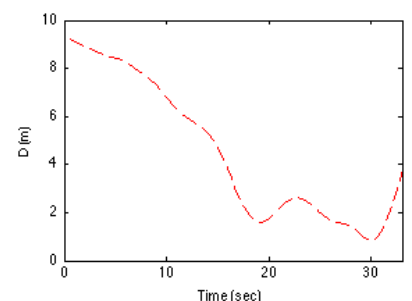

b)
Fig. 9. Experiment 2, run 1. a) shows the evasive trajectory of the vehicle and the location of the target (local tangent plane). b) shows the evolution of $L$ with a minimum value of $0.8 \mathrm{mts}$

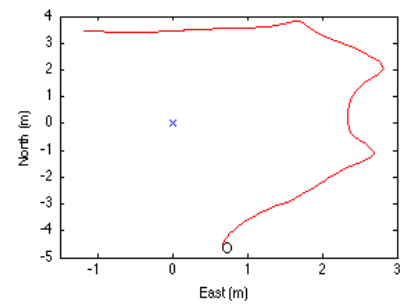

a)

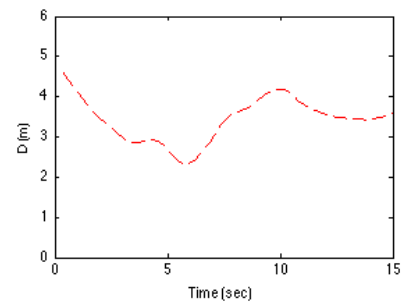

b)
Fig. 10. Experiment 4, run 7.a) shows the evasive trajectory of the vehicle and the location of the target (local tangent plane). b) shows the evolution of $\mathrm{L}$ with a minimum value of $2.35 \mathrm{mts}$

work has been supported by the European Commission and the Australian Academy of Science through a FP7-PEOPLEIRSES-2008 grant (PIRSES-GA-2009-230797 - ICPUAS). The authors also would like to thank Universidad Politécnica de Madrid, Consejería de Educación de la Comunidad de Madrid and Fondo Social Europeo (FSE). The authors acknowledge the contribution and support from Troy Bruggemann, Miguel Olivares Mendez and Carol Martinez.

\section{REFERENCES}

[1] N. Michael, D. Mellinger, Q. Lindsey, and V. Kumar, “The grasp multiple micro-uav testbed," Robotics Automation Magazine, IEEE, vol. 17, no. 3, pp. $56-65,2010$.

[2] I. Dryanovski, W. Morris, and Jizhong Xiao, "Multi-volume occupancy grids: An efficient probabilistic 3d mapping model for micro aerial vehicles," in Intelligent Robots and Systems (IROS), 2010 IEEE/RSJ International Conference on, 2010, pp. 1553 -1559.

[3] S. Lupashin, A. Schö andllig, M. Sherback, and R. D’Andrea, "A simple learning strategy for high-speed quadrocopter multi-flips," in Robotics and Automation (ICRA), 2010 IEEE International Conference on, May 2010, pp. $1642-1648$.

[4] ASTM, "F2411-07 - standard specification for design and performance of an airborne sense-and-avoid system," 2007.

[5] US-OSoD, Unmanned systems roadmap 2009-2034, Office of the Secretary of Defense, Washington, District of Columbia, fourth edition, 2009.

[6] B.C. Karhoff, J.I. Limb, S.W. Oravsky, and A.D. Shephard, "Eyes in the domestic sky: An assessment of sense and avoid technology for the army's warrior unmanned aerial vehicle," in IEEE Systems and Information Engineering Design Symp., April 2006, pp. 36-42.

[7] O. Shakernia, W. Z. Chen, and V. M. Raska, "Passive ranging for uav sense and avoid applications," in Proc. AIAA Infotech@Aerospace Conf., Arlington, Virginia, 2005, pp. 1-10.

[8] Johan Driessen, "Object tracking in a computer vision based autonomous see-and-avoid system for unmanned aerial vehicles," M.S. thesis, Department of Numerical Analysis and Computer Science. Royal Institute of Technology, Stockholm, Sweden, 2004.

[9] Giancarmine Fasano, Multisensor based fully autonomous noncooperative collision avoidance system for UAVs, Phd thesis, Univ. of Naples, 2008.
[10] Jeffrey Saunders and Randal Beard, "Vision-based reactive multiple obstacle avoidance for micro air vehicles," in 2009 American Control Conference, 2009, pp. 5253-5258.

[11] Y. Watanabe, A. J. Calise, and E. N. Johnson, "Vision-based obstacle avoidance for uavs," in Proc. AIAA Guidance, Navigation and Control Conf., Hilton Head, South Carolina, 2007, pp. 20-23.

[12] P. Angelov, C. D. Bocaniala, C. Xideas, C. Patchett, D. Ansell, M. Everett, and G. Leng, "A passive approach to autonomous collision detection and avoidance," in Proc. 10th Int. Conf. on Computer Modeling and Sim., Cambridge, U.K., 2008, pp. 64-69.

[13] Luis Mejias, Scott Mcnamara, John Lai, and Jason J. Ford, "Visionbased detection and tracking of aerial targets for uav collision avoidance," in Proceedings of the 2010 IEEE/RSJ International Conference on Intelligent Robots and Systems (IROS), October 2010, pp. 87-92.

[14] J. Lai, L. Mejias, and J. J. Ford, "Airborne vision-based collisiondetection system," Journal of Field Robotics, vol. 28, no. 2, pp. $137-$ 157, March/April 2011.

[15] P.I. Corke and S.A. Hutchinson, "A new partitioned approach to image-based visual servo control," Robotics and Automation, IEEE Transactions on, vol. 17, no. 4, pp. 507 -515, Aug. 2001.

[16] P.I. Corke, "Spherical image-based visual servo and structure estimation," in Robotics and Automation (ICRA), 2010 IEEE International Conference on, May 2010, pp. 5550-5555.

[17] F. Chaumette and S. Hutchinson, "Visual servo control. i. basic approaches," Robotics Automation Magazine, IEEE, vol. 13, no. 4, pp. $82-90$, dec. 2006.

[18] F. Chaumette and S. Hutchinson, "Visual servo control. ii. advanced approaches [tutorial]," Robotics Automation Magazine, IEEE, vol. 14, no. 1, pp. 109 -118, march 2007.

[19] Christoph Baumgarten and Gerald Farin, "Approximation of logarithmic spirals," Computer Aided Geometric Design, vol. 14, no. 6, pp. 515 - 532, 1997.

[20] Ashley Tews, Jonathan Roberts, and Kane Usher, "Is the sun too bright in queensland? an approach to robust outdoor colour beacon detection," in Australasian Conference on Robotics and Automation (ACRA), 2005.

[21] Gary R. Bradski, "Computer vision face tracking for use in a perceptual user interface," Intel technology Journal, , no. No. Q2, 1998.

[22] Simon Baker and Shree K. Nayar, "A theory of single-viewpoint catadioptric image formation," International Journal of Computer Vision, vol. 35, no. 2, pp. 1-22, 1999.

[23] Christopher Geyer and Konstantinos Daniilidis, "A unifying theory for central panoramic systems and practical applications," in ECCV (2), 2000, pp. 445-461.

[24] Xianghua Ying and Zhanyi Hu, "Can we consider central catadioptric cameras and fisheye cameras within a unified imaging model," in Computer Vision - ECCV 2004, vol. 3021 of Lecture Notes in Computer Science, pp. 442-455. Springer Berlin / Heidelberg, 2004.

[25] João. Barreto and Helder Araujo, "Issues on the geometry of centra catadioptric image formation," Computer Vision and Pattern Recognition, 2001. CVPR 2001. Proceedings of the 2001 IEEE Computer Society Conference on, vol. 2, pp. II-422-II-427 vol.2, 2001.

[26] C. Mei and P. Rives, "Single view point omnidirectional camera calibration from planar grids," in IEEE International Conference on Robotics and Automation, April 2007.

[27] David Austin and Nick Banes, "Red is the new black - or is it?," in Australasian Conference on Robotics and Automation (ACRA), 2003.

[28] K. Fukunaga and L. Hostetler, "The estimation of the gradient of a density function, with applications in pattern recognition," Information Theory, IEEE Transactions on, vol. 21, no. 1, pp. 32 - 40, Jan. 1975.

[29] Luis Mejias, Jason Ford, and John Lai, "Towards the implementation of vision-based uas sense-and-avoid," in Proceedings of the 27th International Congress of the Aeronautical Sciences (ICAS 2010 CDRom), 2010.

[30] S. Benhimane and E. Malis, "A new approach to vision-based robot control with omni-directional cameras," in Robotics and Automation, 2006. ICRA 2006. Proceedings 2006 IEEE International Conference on, May 2006, pp. $526-531$.

[31] S. Hutchinson, G.D. Hager, and P.I. Corke, "A tutorial on visual servo control," Robotics and Automation, IEEE Transactions on, vol. 12, no. 5, pp. $651-670$, Oct. 1996.

[32] CVG-UPM, "Universidad Politécnica de Madrid. Computer Vision Group. Vision for UAV Project," http://www.vision4uav.com, 2010.

[33] "Ascending technologies," http://www.asctec.de, 2010. 